\title{
Incidence of liver disease in people with HFE mutations
}

\author{
G Willis, J Z Wimperis, R Lonsdale, I W Fellows, M A Watson, L M Skipper, B A Jennings
}

\begin{abstract}
Background-Most patients with haemochromatosis have mutations of the HFE gene. However, the risk to people with HFE mutations of developing disease manifestations of haemochromatosis is not known.

Aims-To determine the risk of developing cirrhosis and liver cancer in individuals with HFE mutations in a population where few people were being treated for haemochromatosis.

Methods-215 archive biopsy specimens of liver cancer $(n=34)$ and cirrhosis $(n=190)$ were retrieved from histology archives. Blood samples from 1000 individuals from the normal population were also collected. DNA was extracted from the biopsy specimens and exons 2 and 4 of the HFE gene were amplified using polymerase chain reaction. The products were analysed for the C282Y (845A) and H63D (187G) mutations.
\end{abstract}

Results-Three $(8.8 \%)$ patients from the liver cancer group were homozygous for the $\mathrm{C} 282$ Y mutation. Five $(2.6 \%)$ patients from the cirrhosis group were homozygous for the C282Y mutation. One case fell in both the liver cancer and cirrhosis groups. C282Y homozygosity was thus significantly more frequent in both groups than in the normal population. These 215 cases are representative of a population of about 250000 over 20 years. During this period we estimate that about 260 births or deaths of C282Y homozygous individuals occurred within this population.

Conclusions-A diagnosis of liver cancer or cirrhosis is rare in the lifetime of individuals from this population who are homozygous for the C282Y mutation (2.5\%) upper $95 \%$ confidence interval (CI) $=8 \%)$. Similarly liver disease is rare among C282Y/H63D compound heterozygotes $(1 \%$; upper $95 \% \mathrm{CI}=3.5 \%)$.

(Gut 2000;46:401-404)

Keywords: haemochromatosis, HLA-H; C282Y; H63D; penetrance

Hereditary haemochromatosis is probably the commonest genetic disorder in whites, with an incidence of up to 1 in $200 .^{1-3}$ It is an autosomal recessive condition in which excess iron is absorbed by the intestine. If untreated, affected individuals may accumulate excess iron over many years of adult life and this causes progressive tissue damage. One of the most common disease manifestations of this tissue damage is cirrhosis that may be followed by liver cancer. Liver disease is the commonest cause of death of patients with hereditary haemochromatosis. ${ }^{4-7}$

The discovery of two hereditary haemochromatosis associated mutations in the HFE (HLA-H) gene $^{8}$ has provided a simple DNA based test for the chromosome 6 linked component of hereditary haemochromatosis. This test is already valuable in confirming a diagnosis of hereditary haemochromatosis, particularly in patients where liver biopsy is contraindicated and in patients where blood iron tests may be unreliable. ${ }^{9} 10$ The high frequency of these mutations ${ }^{238}$ and biochemical iron overload ${ }^{1}$ among white populations has led to the suggestion that normal population screening may be justified by health benefits and subsequent healthcare cost savings. ${ }^{11}{ }^{12}$

The majority of people who are homozygous for the C282Y mutation of HFE are not currently being treated by phlebotomy in this region of England ${ }^{3}$ or on the island of Jersey. ${ }^{13}$ This suggested that homozygosity for the C282Y mutation might not always lead to the development of disease. The application of the DNA based test to archive biopsy material has allowed us to carry out retrospective studies of hereditary haemochromatosis associated disease epidemiology in patients who may have been dead for up to 20 years. ${ }^{14}$

\section{Materials and methods}

LIVER DISEASE PATIENTS

The work was approved by the Norwich District Ethics Committee (ref. 97/090). Cases of liver cancer and cirrhosis of the liver were identified from hospital records. Numbers of liver cancer cases were confirmed from Cancer Registry data. ${ }^{15}$ The Norfolk and Norwich Hospital is a large district general hospital and as such is the only referral centre for these diseases in this region; it has a catchment area of 500000 people. The number of liver disease cases seen at this hospital during the period 1976-1996 was: hepatocellular carcinoma (HCC), 49; other primary liver cancers, 22; cirrhosis, 360. Archive biopsy specimens were retrieved; DNA was extracted and amplified from 28 cases of HCC, six cases of other liver cancers (average age 64,72\% men), and 190 cases of cirrhosis (average age 57, 43\% men); nine cases fell in both the HCC and cirrhosis groups.

Reasons for non-inclusion were: not biopsied (estimated to be $10 \%$ of total by the gastroenterology department), wax block no longer

Abbreviations used in this paper: HCC, hepatocellular carcinoma. 
available or empty (18\%), polymerase chain reaction (PCR) amplification failure (16\%), other reasons $(6 \%)$. Therefore, $50 \%$ of known liver disease cases were not available for sampling owing to a variety of independent factors. The samples genotyped are therefore an essentially random sample of half the cases derived from a population of about 500000 people during a 20 year period. The sample is thus representative of a normal population of about 250 000. All liver cancer cases were reviewed histologically.

ARCHIVE BIOPSY DNA EXTRACTION

The surface of the wax block was cleaned by removing a thin layer with a scalpel. A clean scalpel was then used to remove about $10 \mathrm{~mm}^{2}$ tissue, $100 \mu \mathrm{m}$ thick. A parallel procedure was carried out on empty wax blocks for use as no DNA controls. DNA was extracted from wax block biopsy specimens by sequential octane and acetone extractions followed by proteinase $\mathrm{K}$ digestion and heat inactivation.

\section{NORMAL POPULATION}

Blood was collected from 1000 individuals from the hospital catchment area not known to have any manifestation of haemochromatosis (average age 59). Significant subsets within this group were: men over 70 years old undergoing full blood counts $(n=350)$; normal volunteers aged 55-64, randomised to the screening arm of a bowel cancer screening trial $(n=373)$; women under 35 years old undergoing full blood counts $(n=191)$; and patients referred for DNA diagnostic tests unrelated to haemochromatosis and for whom the diagnosis was negative $(n=86)$. All normal group samples were checked to avoid overlap with diagnosed haemochromatosis patients, and age, sex, and any possibly relevant clinical details were recorded. All subsequent testing of normals was anonymous. Thirty $\mathrm{C} 282 \mathrm{Y}$ homozygotes and no $\mathrm{C} 282 \mathrm{Y} / \mathrm{H} 63 \mathrm{D}$ compound heterozygotes from the hospital catchment area are currently being treated by phlebotomy. Individuals with these genotypes are thus estimated to be $98.6 \%$ and $100 \%$ respectively untreated within this population at present. $^{3}$

DNA was extracted from whole blood by standard methods.

DNA AMPLIFICATION

The amino acid 282 region of the HFE gene from archive samples was amplified using nested PCR with short amplimer primers. Blood DNA was amplified using the standard

Table 1 Features of C282Y homozygotes

\begin{tabular}{lllll}
\hline & HH related diagnosis & Liver iron & Age when biopsied & Sex \\
\hline 1 & Undifferentiated liver cancer & NA $^{\star}$ & 60 & $\mathrm{M}$ \\
2 & HCC & NA & 66 & $\mathrm{M}$ \\
3 & HCC/cirrhosis & Grade 1† & 70 & $\mathrm{M}$ \\
4 & Cirrhosis/HH & Grade 3 & 51 & $\mathrm{M}$ \\
5 & Cirrhosis/HH/diabetes & Grade 4 & 58 & $\mathrm{M}$ \\
6 & Cirrhosis/HH/diabetes & Grade 4 & 66 & $\mathrm{M}$ \\
7 & Cirrhosis/HH & Grade 4 & 73 & \\
\hline
\end{tabular}

Cases 1-7 are from 215 patients with liver disease.

${ }^{\star}$ Not applicable; only tumour was present in the needle biopsy sample.

†Minute area of cirrhotic liver.

$\mathrm{HH}$, hereditary haemochromatosis; HCC, hepatocellular carcinoma. primers and method described by Feder and colleagues $^{8}$ and Worwood et al. ${ }^{16}$ No DNA controls comprised $10 \%$ of each batch of samples amplified. All PCR work was carried out in rooms dedicated to either preparation or product analysis.

ALLELE SPECIFIC OLIGONUCLEOTIDE

HYBRIDISATION FOR AMINO ACID 282 ALLELES

Short amplimer PCR products generated from the archive DNA were analysed by base specific oligonucleotide hybridisation. A ${ }^{32} \mathrm{P}$ end labelled oligonucleotide probe specific for either the wild type or mutant allele was used.

RESTRICTION DIGESTION ANALYSIS

PCR products were analysed by digestion with RsaI (282 region) or MboI (63 region) according to the manufacturer's instructions, and electrophoresis.

STATISTICS

Probability values and confidence intervals (CI) were calculated using the normal and Poisson approximations to the binomial distribution and appropriate tables. The confidence interval for the penetrance calculation is derived from the product of the standard errors of the normal population and the disease group homozygote frequencies.

\section{Results}

Three of the $34(8.8 \%)$ cases of liver cancer examined were in patients homozygous for the HFE C282Y mutation. Two of these patients had hepatocellular carcinoma and one had an undifferentiated liver cancer. Five of the 190 $(2.6 \%)$ cases of cirrhosis examined were in patients homozygous for the HFE C282Y mutation. None of the patients with liver cancer was diagnosed with hereditary haemochromatosis before our retrospective analysis. Four of the five patients with cirrhosis were diagnosed with hereditary haemochromatosis at the time of the biopsy used in our analysis (table 1): the remaining case also fell in the liver cancer group. Only one of these seven C282Y homozygotes is alive. Homozygosity has been confirmed from a blood sample in this case; in a further case a homozygous sibling has been identified. No amplification was observed in any no DNA control.

Among the normal population tested, 132 of 2000 chromosomes carried the C282Y mutation (allele frequency $=6.6 \% ; 95 \% \mathrm{CI}=5.5 \%$ to $7.7 \%$ ) and 84 of 600 carried the $187 \mathrm{G}$ mutation (allele frequency $=14 \% ; 95 \% \mathrm{CI}=$ $11.2 \%$ to $16.8 \%$ ). From this we calculate the frequency of C282Y homozygotes in the population to be 1 in 230 and C282Y/H63D compound heterozygotes to be 1 in 108 .

Thus the prevalence of C282Y homozygosity in liver cancer $(\mathrm{p}<0.001)$ and cirrhosis $(p<0.001)$ are both significantly higher than the prevalence in the normal population.

The prevalence of $\mathrm{C} 282 \mathrm{Y} / 282 \mathrm{C}$ heterozygosity did not differ significantly between the liver cancer group (4/34), cirrhosis group $(22 / 190)$, and the normal group (87/700). No 
subset of the normal group had any significant difference in C282Y/282C heterozygote frequency.

None of the 34 cases $(0 \%)$ of liver cancer and six of the 190 cases $(3.1 \%)$ of cirrhosis examined were in patients who were $\mathrm{C} 282 \mathrm{Y} /$ H63D compound heterozygotes. The prevalence of this genotype is significantly higher in the cirrhosis group than that in the normal population $(p<0.005)$. None of these C282Y/ H63D patients had been diagnosed with hereditary haemochromatosis.

The birth and death rate in the population of 250000 from which our liver disease cases are drawn is about 3000 per year. ${ }^{17}$ We calculate that 13 (95\% CI $=9$ to 18$)$ of these individuals per year will be C282Y homozygotes. Seven C282Y homozygous patients were diagnosed with liver disease during the 20 year period for which we have collected samples $(0.35$ per year). Thus only $2.7 \%$ (upper $95 \% \mathrm{CI}=8 \%$ ) of the C282Y homozygotes born each year are diagnosed with liver disease. By a similar calculation we estimate $1 \%$ (upper $95 \% \mathrm{CI}=$ $3.5 \%$ ) of $\mathrm{C} 282 \mathrm{Y} / \mathrm{H} 63 \mathrm{D}$ compound heterozygotes are diagnosed with liver disease.

\section{Discussion}

The discovery of the HFE gene has provided diagnostic molecular genetics with one of the most attractive tests for population screening, because HFE mutations are common and easily detected, and a treatment for hereditary haemochromatosis already exists. ${ }^{18}$ However, although HFE mutations are strongly associated with hereditary haemochromatosis, the association between HFE mutations and the disease manifestations of hereditary haemochromatosis is unclear. ${ }^{13} 19$ The fact that women are diagnosed with haemochromatosis much less frequently than men $^{5-7}$ has provided a basis for the expectation that not every person homozygous for HFE mutations will develop symptomatic disease. We have chosen to address this issue further by a retrospective population based analysis. This method bypasses ethical problems that would arise in withholding treatment during follow up studies of discovered C282Y homozygotes, allows the study of a population much larger than would be feasible in a screening experiment, and yields immediate results.

Our estimate of the proportion of untreated HFE C282Y homozygotes and C282Y/H63D compound heterozygotes developing liver disease in this population is low (less than 5\%) and if this estimate correlates with penetrance in other populations, it would have important implications in several areas of hereditary haemochromatosis management. Firstly, the value of whole population screening will be less than expected. ${ }^{112}$ Secondly, asymptomatic C282Y homozygotes and C282Y/H63D compound heterozygotes may currently be receiving unnecessary treatment. Thirdly, additional factors that determine the disease outcome of untreated HFE C282Y homozygotes and C282Y/H63D compound heterozygotes must be identified.
Liver disease accounts for about half the presenting symptoms of hereditary haemochromatosis and about half the deaths of patients with hereditary haemochromatosis. ${ }^{4-7}$ If this pattern is also true in this population our estimate of the penetrance of the C282Y genotype with respect to all disease manifestations would be about $5 \%$.

The prevalence of $\mathrm{C} 282 \mathrm{Y} / \mathrm{H} 63 \mathrm{D}$ compound heterozygotes in the cirrhosis group was significantly above that predicted from the normal population. However, only one of the six cases with this genotype had stainable iron, classified as grade 1 haemosiderosis, in the biopsied liver. The C282Y/H63D genotype is common in the normal population and so it is not unlikely $(p>0.05)$ that five of these six individuals occurred in the liver disease group by chance. This would be consistent with the observation that the $\mathrm{C} 282 \mathrm{Y} / \mathrm{H} 63 \mathrm{D}$ genotype is significant in hereditary haemochromatosis but is less penetrant than the C282Y homozygous genotype. $^{820}$

Our experimental design is subject to several sources of error as a means of determining penetrance. Firstly, some cases of liver disease may have been left out of the calculation because they were not diagnosed; however, these cases are unlikely to include life threatening cirrhosis or liver cancer, all cases of which are notified to the Cancer Registry. ${ }^{15}$ Secondly, the normal population may not be representative of the disease parent population because of demographic changes; however, the Norfolk, UK population is noted for its stability. ${ }^{21}$ Furthermore, the large majority of recent immigrants come from within the UK where similar frequencies of HFE alleles have been observed. $^{22}$ Thirdly, unaffected C282Y homozygotes may occur in the disease group by chance; we would expect one such individual among the 215 liver disease cases examined, but this does not lower the upper confidence interval for penetrance significantly. All these sources of error are expected to be small compared with the statistical standard error derived from the sample sizes which gives an upper $95 \%$ confidence interval of more than double the estimate of $2.7 \%$. This figure is consistent with a low frequency of deaths attributed to haemochromatosis in the $\mathrm{USA}^{23}$ and our observation that C282Y homozygous men can survive to extreme old age without treatment for haemochromatosis. ${ }^{24}$

The ratio of cases of C282Y homozygotes with cirrhosis to those with liver cancer in our sample is similar to the ratios seen in long term follow up of patients with hereditary haemochromatosis. ${ }^{56}$ This suggests that the disease progression following hereditary haemochromatosis induced cirrhosis is similar in this population to others.

A similarly low estimate of the proportion of C282Y homozygotes with abnormal liver function results was suggested by the examination of a large sample of patients with raised serum alanine aminotransferase. ${ }^{25}$ Low penetrance of the C282Y homozygous genotype in the British population may be unusual and may explain the small number of patients currently 
being treated for hereditary haemochromatosis at this hospital. ${ }^{3}$ Various factors could account for low penetrance. Genes not linked to HFE could affect iron absorption, patterns of storage, ${ }^{26}$ and tolerance of iron overload in tissues. The existence of genetic variation in these functions is suggested by the diverse manifestations of iron overload and differences between individuals with hereditary haemochromatosis and secondary haemochromatosis. Diet is also likely to be an important factor.

It is now important to determine whether the C282Y homozygotes in this population who do not develop hereditary haemochromatosis related liver disease, do not accumulate large iron stores or whether they tolerate high iron stores. It is also important to determine whether this pattern of liver disease among C282Y homozygotes occurs in other populations.

1 Edwards CQ, Griffen LM, Goldgar D, et al. Prevalence of hemochromatosis among 11,065 presumably healthy blood donors. N Engl f Med 1988;318:1355-62.

2 Merryweather-Clarke AT, Pointon JJ, Shearman JD, et al. Global prevalence of putative haemochromatosis mutaGlobal prevalence of putative haem
tions. $\mathcal{F}$ Med Genet $1997 ; 34: 275-8$.

3 Willis G, Jennings BA, Goodman E, et al. A high prevalence of HLA-H 845A mutations in hemochromatosis patient and the normal population in eastern England. Blood Cell Mol Dis 1997;23:288-91.

4 Milder MS, Cook JD, Stray S, et al. Idiopathic hemochromatosis, an interim report. Medicine 1980;59:34-49.

5 Niederau C, Fischer R, Purschel A, et al. Long term survival in patients with hereditary hemochromatosis. Gastroenterology 1996;110:1107-19.

6 Adams PC, Speechley M, Kertesz AE. Long-term survival analysis in hereditary hemochromatosis. Gastroenterology 1991;101:368-72.

7 Fargion S, Mandelli C, Piperno A, et al. Survival and prognostic factors in 212 Italian patients with genetic hemochromatosis. Hepatology 1992;15:655-9.

8 Feder JN, Gnirke A, Thomas W, et al. A novel MHC class I-like gene is mutated in patients with hereditary I-like gene is mutated in patients with

9 Cotler SJ, Bronner MP, Press RD, et al. End-stage liver disease without hemochromatosis associated with elevated hepatic iron index. F Hepatol 1998;29:257-62.
10 Adams PC, Chakrabarti S. Genotypic/phenotypic correlations in genetic hemochromatosis: evolution of diagnostic criteria. Gastroenterology 1998;114:319-23.

11 Bassett ML, Leggett BA, Halliday JW, et al. Analysis of the cost of population screening for haemochromatosis using biochemical and genetic markers. Hepatology 1997;27:51724.

12 Burt MJ, George PM, Upton JD, et al. The significance of haemochromatosis gene mutations in the general population: implications for screening. Gut 1998;43:830-6.

13 Merryweather-Clarke AT, Worwood M, Parkinson L, et al. The effect of HFE mutations on serum ferritin and transferrin saturation in the Jersey population. Br f Haematol 1998;101:369-73.

14 Willis G, Wimperis JZ, Lonsdale R, et al. Haemochromatosis gene mutation in hepatocellular cancer. Lancet 1997; 350:565-6.

15 Davies TW. A report on cancer incidence in 1994. Cambridge, UK: East Anglian Cancer Registry, 1995.

16 The UK Haemochromatosis Consortium. A simple genetic test identifies $90 \%$ of UK patients with haemochromatosis. Gut 1997;41:841-4.

17 Barker M. Health and personal social services statistics for England. London, UK: HMSO, 1995.

18 Bomford A, Williams R. Long term results of venesection therapy in idiopathic haemochromatosis. $Q 7$ Med 1976;45: 611-23.

19 Burke W, Thompson E, Khoury MJ, et al. Hereditary hemochromatosis: gene discovery and its implications for population screening. $\mathcal{F A M A}$ 1998;280:172-8.

20 Beutler E, Gelbart T, West C, et al. Mutation analysis in hereditary hemochromatosis. Blood Cells Mol Dis 1996;22: 187-94.

21 Symmons PM, Barrett EM, Bankhead CR, et al. The incidence of rheumatoid arthritis in the United Kingdom: results from the Norfolk arthritis register. Br $\mathcal{F}$ Rheumatol 1994;33:735-9.

22 Frayling T, Elard S, Grove J, et al. C282Y mutation in HFE (haemochromatosis) gene and type 2 diabetes. Lancet 1998;351:1933-4.

23 Yang Q, McDonnell SM, Khoury MJ, et al. Hemochromatosis-associated mortality in the United States from 1979 to 1992: an analysis of multiple case mortality data. Ann Intern Med 1998;129:946-53.

24 Willis G, Wimperis JZ, Smith KC, et al. HFE (haemochromatosis gene) $\mathrm{C} 282 \mathrm{Y}$ homozygotes in an elderly male population. Lancet 1999;354:221-2.

25 Bhavnani M, Bhattacharyya A, Lloyd D, et al. Prevalence of genetic haemochromatosis $(\mathrm{GH})$ in patients with raised serum alanine aminotransferase. Blood 1998;92:1329a.

26 Crawford DHG, Halliday JW, Summers KM, et al. Concordance of iron storage in siblings with genetic hemochromatosis: evidence for a predominantly genetic effect on iron storage. Hepatology 1993;17:833-7. 